\title{
LA MEDIDA DE LAS ACTITUDES USANDO LAS TÉCNICAS DE LIKERT Y DE DIFERENCIAL SEMÁNTICO
}

\author{
ESPINOSA GARCÍA, J. ${ }^{1}$ y ROMÁN GALÁN, T. ${ }^{2}$ \\ ${ }^{1}$ Departamento de Química Física. Facultad de Ciencias. Universidad de Extremadura. 06071 Badajoz. \\ ${ }^{2}$ IES Pedro de Valdivia.Villanueva de la Serena. 06700 Badajoz.
}

\section{SUMMARY}

We have established a comparison between two popular methods to assess the attitude toward experimental sciences: The summated rating method, generally known as the Likert scale; and the semantic differential method. This study is carried out with university students of chemistry and high school students, finding that both attitude measuring techniques are equally suitable in our educative system. Moreover, the sex variable is not significant.

\section{INTRODUCCIÓN}

Cuando usamos una determinada técnica para medir las actitudes de los estudiantes hacia cualquier objeto, en nuestro caso particular la ciencia experimental, podemos plantearnos si realmente estamos midiendo este aspecto afectivo de la enseñanza tan difícilmente cuantificable, y en qué medida los resultados obtenidos son dependientes de la técnica. Entre las muchas técnicas de medida desarrolladas a lo largo del tiempo, dos han sido las más populares: el método de clases sumadas (summated rating scale), generalmente conocido como escala de Likert, y el método de diferencial semántico (DS). Recientemente, se ha prestado mucha atención a la validación de los cuestionarios, proceso de selección y consistencia interna, destacando los trabajos de Simpson y Oliver (1985) (escala de Likert con 7 ítems); Germann (1988) (escala de Likert con 14 ítems) y Misisti y sus colaboradores (1991) (escala de Likert con 23 ítems). Una de las ventajas adicionales del último instrumento de medida es que ha superado con éxito uno de los problemas de estos cuestionarios, es decir, la traducción a otra lengua diferente de la original. En efecto, Mejías (1988) (citado por Misiti y col.) tradujo al castellano la anterior escala (con pequeñas modificaciones) procediendo a su validación. Misiti y sus colaboradores concluyen que su escala tiene algún grado de validez transcultural cuando se traduce al castellano.

A pesar de la popularidad de estas dos técnicas (Likert y diferencial semántico) y el uso extendido de los diferentes intrumentos de medida, varios trabajos han puesto de manifiesto las dificultades de la medición de las actitudes, especialmente usando la técnica de Likert (Munby, 1983; Shrigley y Koballa, 1984). La afirmación del profesor Munby de que la validez conceptual del «inventario de actitudes científicas» es, al menos, sospechoso originó controversias (Zeidler, 1984; Bratt, 1984) y recomendaciones generales sobre cómo medir las actitudes de nuestros alumnos (Henerson, 1985). 
Una cuestión aún abierta es si los resultados obtenidos con diferentes métodos son equiparables. Desafortunadamente, existen muy pocos trabajos que comparen ambas técnicas (Likert o DS) y, según nuestro conocimiento, ninguno en el sistema educativo español. Entre estos pocos trabajos, McCallon y Braun (1971) encuentran una alta correlación positiva $(r=0,90)$ entre ambas técnicas cuando evalúan las actitudes hacia las matemáticas; Schofield y Start (1978) evalúan también la actitud hacia las matemáticas con ambas técnicas y encuentran una moderadamente alta correlación $(\mathrm{r}=0,70)$; $\mathrm{y}$ Schibeci (1982) evalúa varios objetos de actitud con ambos métodos, encontrando en todos los casos una correlación media $(r<0,52)$.

Los dos primeros trabajos pertenecen a alumnos universitarios (college), mientras que el último corresponde a alumnos de enseñanza secundaria (12-17 años, high school). Para estos últimos estudiantes, Schibeci concluye que ambas técnicas son difícilmente intercambiables y que, mientras la técnica de diferencial semántico permite una rápida medida de la actitudes generales hacia la ciencia, cuando deseamos evaluar actitudes más específicas es recomendable la técnica de Likert. En conclusión, como puede verse, existe una falta de información al respecto con disparidad de resultados, y ésta parece ser aún una cuestión abierta.

En este trabajo presentamos los resultados de alumnos de cuarto de ESO (15-16 años) y de tercero de Ciencias Químicas (20-21 años) usando las técnicas de Likert y de diferencial semántico. Pensamos que se trata de un trabajo pionero en nuestro sistema educativo y que viene a completar la investigación que nuestro grupo está desarrollando sobre el análisis de las actitudes de los alumnos hacia la ciencia (Espinosa y Román, 1991; Espinosa y Román, 1993; Espinosa y Román, 1995; Armas, Espinosa y Román, 1996).

\section{DISEÑO DE LA EXPERIENCIA. INSTRU- MENTOS DE MEDIDA, MUESTRA Y OBJETIVOS}

Como instrumentos de medida usamos los cuestionarios de diferencial semántico y de Likert. En el primero, usamos un cuestionario ampliamente utilizado por nosotros en anteriores trabajos, donde se plantean 10 cuestiones contrarias que el alumno debe puntuar de 0 a 10 (Anexo I). Este cuestionario se ha mostrado rápido y fiable así como bastante familiar para el alumno. El encabezamiento del cuestionario usado en este trabajo estaba orientado hacia los estudios de ciencias experimentales, sin concretar disciplina. Aunque es cierto que las actitudes de los alumnos hacia la física y la química son diferentes que hacia la biología y la geología (Acevedo, 1993), el objetivo de este trabajo era analizar la actitud hacia las ciencias experimentales en general, como una idea globalizadora. Como cuestionario de Likert elegimos el propuesto por Misiti y sus colabora- dores, que presenta la enorme ventaja (con el consiguiente ahorro de tiempo) de haber superado la validación transcultural cuando se tradujo al castellano (Mejías, 1988). Debido a la redundancia de muchas de las cuestiones y con el objetivo de hacer más clara la exposición a los alumnos, la redacción final contiene sólo 15 de las 23 cuestiones originales, y la elaboración de la escala definitiva tuvo en cuenta las recomendaciones de Edward (1957) y Henerson (1985). En general, la mitad de las cuestiones debe presentar una actitud positiva y la otra mitad, negativa; el lenguaje debe ser coloquial y comprensible para el alumno, evitando todo tecnicismo; y palabras tales como todo o nada deben ser usadas con cuidado. En el cuestionario del presente trabajo se plantean 15 cuestiones con algún enunciado sobre el objeto de actitud que se desea medir, como, por ejemplo, «Comprar libros de ciencias es pesadísimo» (Anexo II). Entonces, se pide al alumno que indique su acuerdo o desacuerdo con cada afirmación en una escala de cinco puntos: totalmente de acuerdo (TA), de acuerdo (A), indeciso (N), en desacuerdo (D) y totalmente en desacuerdo (TD). La ventaja de este instrumento de medida es que el sistema de preguntas es muy familiar para el alumno y abarca ampliamente el objeto de actitud a medir.

La encuesta fue realizada a principios del curso 1995-96 con 180 alumnos ( 81 chicos y 99 chicas) de $4^{\circ}$ de ESO del IES Pedro de Valdivia de Villanueva de la Serena (Badajoz), como muestra de la enseñanza secundaria; y con 60 alumnos (20 chicos y 40 chicas) de $3^{\circ}$ de Ciencias Químicas de la Universidad de Extremadura, como muestra de enseñanza universitaria. El intervalo entre ambos cuestionarios (Likert y diferencial semántico) fue de tan sólo una semana, para evitar perturbaciones en los resultados por otros factores no considerados en este trabajo.

Nuestras hipótesis de trabajo son las siguientes:

a) Poner de manifiesto, por primera vez en nuestro sistema educativo, si las técnicas de Likert y de diferencial semántico pueden medir de forma equiparable la actitud de los alumnos hacia la ciencia.

b) Analizar cualitativamente si la variable sexo es significativa.

c) Analizar si las diferencias encontradas en los países anglosajones entre ambas técnicas para la enseñanza secundaria y universitaria se siguen manteniendo en nuestro sistema educativo.

\section{RESULTADOS Y DISCUSIÓN}

En las tablas I y II se presentan los resultados para estudiantes universitarios de $3^{\circ}$ de Químicas, usando los cuestionarios de diferencial semántico y de Likert, respectivamente; y en las tablas IV y V, los correspondientes a alumnos de secundaria de $4^{\circ}$ de ESO. 


\section{Alumnos universitarios}

Con el cuestionario de diferencial semántico (Tabla I), los valores medios de cada cuestión indican una clara actitud positiva hacia las ciencias experimentales, y el análisis de cada cuestión individual nos indica que las experiencia acumulada por los alumnos universitarios durante los años de carrera les hace difíciles y liosas las ciencias experimentales (cuestiones 2 y 3 ) aunque las encuentren interesantes (cuestiones 1, 5, 8 y 10). Con respecto a la variable sexo, debemos indicar que cualitativamente no hay diferencias significativas, encontrándose las mayores diferencias en las cuestiones 2 y 3 . Las chicas encuentran las ciencias experimentales ligeramente más liosas y difíciles que los chicos.

Los resultados con la técnica de Likert se recogen en la tabla II, donde se comparan los resultados de la actitud de chicos, chicas y totales para cada una de las cuestiones del anexo II. Los valores finales de los chicos $(+33 \%$ primera fila en cada cuestión), de las chicas $(+38 \%$ segunda fila en cada cuestión) y totales, $(+36 \%$, tercera fila en cada cuestión), indican una clara actitud positiva hacia las ciencias experimentales. Estos resultados concuerdan con los obtenidos usando la técnica de diferencial semántico. El análisis de cada cuestión individual nos indica que, excepto la cuestión 4 , que presenta una actitud muy negativa, y las cuestiones 2,6 y 11 , que presentan una actitud prácticamente neutra, la actitud es marcadamente positiva. Con respecto a la variable sexo, cualitativamente encontramos que no hay diferencias significativas y vemos las mayores diferencias en las cuestiones 2 y 6 , donde los chicos tienen una actitud más negativa.

En conclusión, hemos comprobado que ambas técnicas para medir las actitudes de los alumnos hacia las ciencias experimentales son equiparables en nuestro sistema educativo universitario, encontrando en ambos casos una clara actitud positiva en chicos y chicas. Como es obvio, cada una de las técnicas (DS y Likert) usa una escala de puntuaciones diferente. Sin embargo, con el objetivo de hacer una comparación más directa entre ambas técnicas, estábamos interesados en homogeneizar los resultados. Para ello, usamos la «escala de puntuaciones» propuesta en trabajos anteriores de nuestro grupo (Espinosa y Román, 1991, 1993 y 1995). Esta escala se muestra particularmente interesante por su simplicidad y eficacia. Así, para la técnica de diferencial semántico, agrupamos en cada cuestión el número de alumnos con puntuaciones 0-3 (actitud negativa), 4-6 (actitud indiferente) y 7-10 (actitud positiva); y, para la técnica de Likert, agrupamos en cada cuestión el número de alumnos con elección D+TD (actitud negativa), $\mathrm{N}$ (indiferente) y A+TA (actitud positiva). Los resultados expresados en tanto por ciento aparecen en la tabla III.

Tabla I

Técnica de diferencial semántico para alumnos universitarios.

\begin{tabular}{|l|c|c|c|c|c|c|}
\cline { 2 - 7 } & \multicolumn{2}{c|}{} & \multicolumn{2}{c|}{ VARONES } & \multicolumn{2}{c|}{ TOTALES } \\
\cline { 2 - 7 } & $\bar{x}$ & $\sigma$ & $\bar{x}$ & $\sigma$ & $\bar{x}$ & \\
\hline 1. Interesante / Aburrido & 9,28 & 0,80 & 9,38 & 0,83 & 9,35 & 0,82 \\
\hline 2. Claro / Lioso & 5,60 & 1,14 & 4,85 & 2,31 & 5,10 & 1,92 \\
\hline 3. Fácil / Difícil & 5,14 & 2,56 & 3,80 & 2,28 & 4,25 & 2,37 \\
\hline 4. Naturaleza / Ni en broma & 6,92 & 2,84 & 6,31 & 2,36 & 6,51 & 2,52 \\
\hline 5. Probaría / Jamás & 5,80 & 2,69 & 8,40 & 1,90 & 8,43 & 2,16 \\
\hline 6. Asequible / Imposible & 6,77 & 1,97 & 7,08 & 2,10 & 6,97 & 2,05 \\
\hline 7. He aprendido / Nada & 9,00 & 1,56 & 8,60 & 1,40 & 8,73 & 1,45 \\
\hline 8. Me ha gustado / Nada & 8,78 & 1,26 & 8,77 & 1,04 & 8,77 & 1,11 \\
\hline 9. Divertido / He sufrido & 6,07 & 2,79 & 6,00 & 2,18 & 6,02 & 2,38 \\
\hline 10. Valía la pena / Tiempo perdido & 8,43 & 2,47 & 9,08 & 1,08 & 8,86 & 1,54 \\
\hline
\end{tabular}


Tabla II

Técnica de Likert para alumnos universitarios.

\begin{tabular}{|c|c|c|c|c|c|c|c|}
\hline CUESTIÓN & $\begin{array}{c}\mathrm{N}^{\circ} \text { de } 1 \\
(-2)\end{array}$ & $\begin{array}{c}\mathrm{N}^{\circ} \text { de } 2 \\
(-1)\end{array}$ & $\begin{array}{c}\mathrm{N}^{\circ} \text { de } 3 \\
(0)\end{array}$ & $\begin{array}{c}\mathrm{N}^{\mathrm{o}} \mathrm{de} \\
(+1)\end{array}$ & $\begin{array}{c}\mathrm{N}^{\circ} \text { de } 5 \\
(+2)\end{array}$ & $\begin{array}{l}\text { TOTAL } \\
\left(+0^{-}\right)\end{array}$ & $\%$ \\
\hline 1 & $\begin{array}{l}3 \\
1 \\
4\end{array}$ & $\begin{array}{l}2 \\
4 \\
6\end{array}$ & $\begin{array}{r}4 \\
8 \\
12\end{array}$ & $\begin{array}{r}8 \\
20 \\
28\end{array}$ & $\begin{array}{r}3 \\
7 \\
10\end{array}$ & $\begin{array}{r}+6 \\
+28 \\
+34\end{array}$ & $\begin{array}{l}+15 \\
+35 \\
+28\end{array}$ \\
\hline 2 & $\begin{array}{l}4 \\
1 \\
5\end{array}$ & $\begin{array}{r}3 \\
9 \\
12\end{array}$ & $\begin{array}{r}5 \\
7 \\
12\end{array}$ & $\begin{array}{r}8 \\
20 \\
28\end{array}$ & $\begin{array}{l}0 \\
3 \\
3\end{array}$ & $\begin{array}{r}-3 \\
+15 \\
+12\end{array}$ & $\begin{array}{r}-8 \\
+19 \\
+10\end{array}$ \\
\hline 3 & $\begin{array}{l}0 \\
0 \\
0\end{array}$ & $\begin{array}{l}0 \\
1 \\
1\end{array}$ & $\begin{array}{r}4 \\
8 \\
12\end{array}$ & $\begin{array}{l}11 \\
16 \\
27\end{array}$ & $\begin{array}{r}5 \\
15 \\
20\end{array}$ & $\begin{array}{l}+21 \\
+45 \\
+66\end{array}$ & $\begin{array}{l}+52 \\
+56 \\
+55\end{array}$ \\
\hline 4 & $\begin{array}{l}13 \\
16 \\
29\end{array}$ & $\begin{array}{r}6 \\
20 \\
26\end{array}$ & $\begin{array}{l}0 \\
3 \\
3\end{array}$ & $\begin{array}{l}0 \\
0 \\
0\end{array}$ & $\begin{array}{l}1 \\
1 \\
2\end{array}$ & $\begin{array}{l}-31 \\
-51 \\
-82\end{array}$ & $\begin{array}{l}-77 \\
-64 \\
-68\end{array}$ \\
\hline 5 & $\begin{array}{l}1 \\
0 \\
1\end{array}$ & $\begin{array}{l}0 \\
0 \\
0\end{array}$ & $\begin{array}{l}1 \\
3 \\
4\end{array}$ & $\begin{array}{l}11 \\
18 \\
29\end{array}$ & $\begin{array}{r}7 \\
19 \\
26\end{array}$ & $\begin{array}{l}+24 \\
+56 \\
+80\end{array}$ & $\begin{array}{l}+60 \\
+70 \\
+66\end{array}$ \\
\hline 6 & $\begin{array}{l}2 \\
0 \\
2\end{array}$ & $\begin{array}{l}1 \\
6 \\
7\end{array}$ & $\begin{array}{l}14 \\
20 \\
34\end{array}$ & $\begin{array}{r}2 \\
12 \\
14\end{array}$ & $\begin{array}{l}1 \\
2 \\
3\end{array}$ & $\begin{array}{r}-1 \\
+10 \\
+9\end{array}$ & $\begin{array}{r}-3 \\
+13 \\
+8\end{array}$ \\
\hline 7 & $\begin{array}{l}1 \\
1 \\
2\end{array}$ & $\begin{array}{l}2 \\
5 \\
7\end{array}$ & $\begin{array}{l}3 \\
5 \\
8\end{array}$ & $\begin{array}{r}9 \\
18 \\
27\end{array}$ & $\begin{array}{r}5 \\
11 \\
16\end{array}$ & $\begin{array}{l}+15 \\
+33 \\
+48\end{array}$ & $\begin{array}{l}+37 \\
+41 \\
+40\end{array}$ \\
\hline 8 & $\begin{array}{l}1 \\
0 \\
1\end{array}$ & $\begin{array}{l}1 \\
3 \\
4\end{array}$ & $\begin{array}{l}10 \\
19 \\
29\end{array}$ & $\begin{array}{r}8 \\
15 \\
23\end{array}$ & $\begin{array}{l}0 \\
3 \\
3\end{array}$ & $\begin{array}{r}+5 \\
+21 \\
+26\end{array}$ & $\begin{array}{l}+12 \\
+26 \\
+22\end{array}$ \\
\hline 9 & $\begin{array}{l}0 \\
0 \\
0\end{array}$ & $\begin{array}{l}0 \\
1 \\
1\end{array}$ & $\begin{array}{l}2 \\
2 \\
4\end{array}$ & $\begin{array}{l}11 \\
29 \\
40\end{array}$ & $\begin{array}{r}7 \\
8 \\
15\end{array}$ & $\begin{array}{l}+25 \\
+44 \\
+69 \\
\end{array}$ & $\begin{array}{l}+62 \\
+55 \\
+57\end{array}$ \\
\hline 10 & $\begin{array}{l}2 \\
0 \\
2\end{array}$ & $\begin{array}{l}0 \\
0 \\
0\end{array}$ & $\begin{array}{l}3 \\
4 \\
7\end{array}$ & $\begin{array}{r}8 \\
23 \\
31\end{array}$ & $\begin{array}{r}7 \\
13 \\
20\end{array}$ & $\begin{array}{l}+18 \\
+49 \\
+67\end{array}$ & $\begin{array}{l}+45 \\
+61 \\
+56\end{array}$ \\
\hline 11 & $\begin{array}{l}3 \\
4 \\
7\end{array}$ & $\begin{array}{l}2 \\
3 \\
5\end{array}$ & $\begin{array}{r}9 \\
18 \\
27\end{array}$ & $\begin{array}{r}3 \\
7 \\
10 \\
\end{array}$ & $\begin{array}{l}3 \\
2 \\
5\end{array}$ & $\begin{array}{r}+1 \\
0 \\
+1 \\
\end{array}$ & $\begin{array}{r}+3 \\
0 \\
+3\end{array}$ \\
\hline 12 & $\begin{array}{l}0 \\
0 \\
0\end{array}$ & $\begin{array}{l}0 \\
0 \\
0\end{array}$ & $\begin{array}{l}2 \\
2 \\
4\end{array}$ & $\begin{array}{r}9 \\
25 \\
34\end{array}$ & $\begin{array}{r}9 \\
13 \\
22\end{array}$ & $\begin{array}{l}+27 \\
+51 \\
+78\end{array}$ & $\begin{array}{l}+67 \\
+64 \\
+65\end{array}$ \\
\hline 13 & $\begin{array}{l}0 \\
0 \\
0\end{array}$ & $\begin{array}{l}0 \\
1 \\
1\end{array}$ & $\begin{array}{l}0 \\
5 \\
5\end{array}$ & $\begin{array}{r}4 \\
13 \\
17\end{array}$ & $\begin{array}{l}16 \\
21 \\
37\end{array}$ & $\begin{array}{l}+36 \\
+33 \\
+69\end{array}$ & $\begin{array}{l}+90 \\
+41 \\
+57\end{array}$ \\
\hline 14 & $\begin{array}{l}0 \\
0 \\
0\end{array}$ & $\begin{array}{l}0 \\
0 \\
0\end{array}$ & $\begin{array}{l}1 \\
0 \\
1\end{array}$ & $\begin{array}{r}9 \\
19 \\
28\end{array}$ & $\begin{array}{l}10 \\
21 \\
31\end{array}$ & $\begin{array}{l}+29 \\
+61 \\
+90\end{array}$ & $\begin{array}{l}+72 \\
+76 \\
+75\end{array}$ \\
\hline 15 & $\begin{array}{l}1 \\
2 \\
3\end{array}$ & $\begin{array}{l}1 \\
3 \\
4\end{array}$ & $\begin{array}{l}0 \\
3 \\
3\end{array}$ & $\begin{array}{l}11 \\
19 \\
30\end{array}$ & $\begin{array}{r}7 \\
13 \\
20\end{array}$ & $\begin{array}{l}+22 \\
+38 \\
+60\end{array}$ & $\begin{array}{l}+55 \\
+47 \\
+50\end{array}$ \\
\hline TOTAL & $\begin{array}{l}31 \\
25 \\
56\end{array}$ & $\begin{array}{l}18 \\
56 \\
74\end{array}$ & $\begin{array}{r}58 \\
117 \\
175\end{array}$ & $\begin{array}{l}112 \\
254 \\
366\end{array}$ & $\begin{array}{r}83 \\
153 \\
236\end{array}$ & $\begin{array}{l}+198 \\
+454 \\
+652\end{array}$ & $\begin{array}{l}+33 \\
+38 \\
+36\end{array}$ \\
\hline
\end{tabular}


Ahora, con esta escala de puntuaciones, común para las dos técnicas, la semejanza de resultados es aún más clara. Con ambas técnicas se encuentra que un $67 \%$ de alumnos tiene una actitud positiva hacia las ciencias experimentales, y alrededor de un $10 \%$ (7\% si usamos la de DS y un $15 \%$ si usamos la de Likert) presenta una actitud negativa. Estos resultados concuerdan en gran medida con los obtenidos por nuestro grupo entre los alumnos del mismo curso de Químicas, pero cuatro promociones anteriores (Espinosa y Román, 1993) reflejan una garantía del instrumento usado para la medida de las actitudes hacia la ciencia experimental.

\section{Alumnos de secundaria}

En la tabla IV se presentan los resultados con la técnica de diferencial semántico, donde los valores medios indican una clara actitud positiva hacia la ciencia. Llama la atención la semejanza con los resultados obtenidos con alumnos universitarios. Así, los alumnos de $4^{\circ}$ de ESO encuentran la ciencia ligeramente difícil y liosa (cuestiones 2 y 3 ), aunque también interesante (cuestiones 1 ,
5,8 y 10). Esta misma tendencia ya fue encontrada por nuestro grupo en un estudio previo con estudiantes de secundaria de $2^{\circ}$ de BUP (Espinosa y Román, 1991). Cuando analizamos la variable sexo, no encontramos diferencias significativas y, en cualquier caso, menores que en estudiantes universitarios (Tabla I).

En la tabla V se recogen los resultados usando la técnica de Likert, donde se comparan los resultados de la actitud de chicos, chicas y totales para cada una de las cuestiones del anexo II. Los valores finales han sido: $+36 \%$, $+35 \%$ y $35 \%$, para chicos, chicas y total, respectivamente, indicando una clara actitud positiva hacia las ciencias experimentales. Una vez más, como en el caso de estudiantes universitarios, ambas técnicas de medida de actitudes proporcionan resultados semejantes. Por otra parte, todas las cuestiones individuales presentan una alta actitud positiva, con diferencias despreciables entre chicos y chicas.

Por tanto, también para estudiantes de enseñanza secundaria hemos comprobado que ambas técnicas son equi-

Tabla III

\begin{tabular}{|l|l|r|r|r|r|r|r|}
\cline { 2 - 9 } \multicolumn{2}{c|}{} & \multicolumn{2}{c|}{ VARONES } & \multicolumn{2}{c|}{ MUJERES } & \multicolumn{2}{c|}{ TOTALES } \\
\hline ESCALA & VALORACIÓN & DS & L & DS & L & DS & L \\
\hline $0-3$ o D+TD & Negativa & 7 & 17 & 8 & 14 & 7 & 15 \\
\hline $4-6$ o N & Indiferente & 22 & 19 & 28 & 18 & 26 & 18 \\
\hline $7-10$ o A+TA & Positiva & 71 & 64 & 64 & 68 & 67 & 67 \\
\hline
\end{tabular}

Tabla IV

Técnica de diferencial semántico para estudiantes de secundaria.

\begin{tabular}{|c|c|c|c|c|c|c|}
\hline & \multicolumn{2}{|c|}{ VARONES } & \multicolumn{2}{|c|}{ MUJERES } & \multicolumn{2}{|c|}{ TOTALES } \\
\hline & $\overline{\mathrm{x}}$ & $\sigma$ & $\overline{\mathrm{x}}$ & $\sigma$ & $\overline{\mathrm{x}}$ & $\sigma$ \\
\hline 1. Interesante / Aburrido & 7,65 & 1,30 & 7,92 & 1,36 & 7,81 & 1,32 \\
\hline 2. Claro / Lioso & 5,32 & 0,95 & 5,71 & 1,18 & 5,51 & 1,11 \\
\hline 3. Fácil / Difícil & 5,61 & 0,93 & 5,41 & 1,12 & 5,50 & 1,07 \\
\hline 4. Naturaleza / Ni en broma & 6,71 & 0,88 & 6,90 & 0,91 & 6,82 & 0,90 \\
\hline 5. Probaría / Jamás & 7,32 & 1,15 & 7,70 & 0,95 & 7,58 & 1,11 \\
\hline 6. Asequible / Imposible & 5,30 & 1,30 & 5,70 & 1,00 & 5,60 & 1,20 \\
\hline 7. He aprendido / Nada & 8,20 & 0,80 & 8,47 & 0,84 & 8,39 & 0,83 \\
\hline 8. Me ha gustado / Nada & 8,71 & 0,90 & 8,90 & 0,66 & 8,82 & 0,71 \\
\hline 9. Divertido / He sufrido & 7,64 & 1,34 & 7,41 & 0,92 & 7,51 & 1,11 \\
\hline 10.Valía la pena / Tiempo perdido & 9,00 & 0,65 & 8,70 & 0,62 & 8,81 & 0,64 \\
\hline
\end{tabular}


Tabla V

Técnica de Likert para estudiantes de secundaria.

\begin{tabular}{|c|c|c|c|c|c|c|c|}
\hline CUESTIÓN & $\begin{array}{c}\mathrm{N}^{\circ} \text { de } 1 \\
(-2)\end{array}$ & $\begin{array}{c}\mathrm{N}^{\circ} \text { de } 2 \\
(-1)\end{array}$ & $\begin{array}{c}\mathrm{N}^{\circ} \text { de } 3 \\
(0)\end{array}$ & $\begin{array}{c}\mathrm{N}^{\circ} \text { de } 4 \\
(+1)\end{array}$ & $\begin{array}{c}\mathrm{N}^{\circ} \text { de } 5 \\
(+2)\end{array}$ & $\begin{array}{r}\text { TOTAL } \\
(+0-)\end{array}$ & $\%$ \\
\hline 1 & $\begin{array}{r}7 \\
11 \\
18\end{array}$ & $\begin{array}{l}3 \\
4 \\
7\end{array}$ & $\begin{array}{l}21 \\
26 \\
47\end{array}$ & $\begin{array}{l}18 \\
21 \\
39\end{array}$ & $\begin{array}{l}30 \\
36 \\
66\end{array}$ & $\begin{array}{r}+61 \\
+67 \\
+128\end{array}$ & $\begin{array}{l}+37 \\
+33 \\
+35\end{array}$ \\
\hline 2 & $\begin{array}{r}8 \\
11 \\
19 \\
\end{array}$ & $\begin{array}{l}4 \\
1 \\
5\end{array}$ & $\begin{array}{l}22 \\
26 \\
48\end{array}$ & $\begin{array}{l}18 \\
21 \\
39 \\
\end{array}$ & $\begin{array}{l}31 \\
37 \\
68 \\
\end{array}$ & $\begin{array}{r}+60 \\
+72 \\
+132 \\
\end{array}$ & $\begin{array}{l}+37 \\
+36 \\
+37\end{array}$ \\
\hline 3 & $\begin{array}{r}8 \\
10 \\
18\end{array}$ & $\begin{array}{l}4 \\
3 \\
7\end{array}$ & $\begin{array}{l}22 \\
26 \\
48\end{array}$ & $\begin{array}{l}18 \\
21 \\
39\end{array}$ & $\begin{array}{l}31 \\
36 \\
67\end{array}$ & $\begin{array}{r}+60 \\
+70 \\
+130\end{array}$ & $\begin{array}{l}+37 \\
+35 \\
+36\end{array}$ \\
\hline 4 & $\begin{array}{r}6 \\
9 \\
15\end{array}$ & $\begin{array}{l}5 \\
3 \\
8\end{array}$ & $\begin{array}{l}22 \\
24 \\
46\end{array}$ & $\begin{array}{l}19 \\
21 \\
40\end{array}$ & $\begin{array}{l}30 \\
36 \\
66\end{array}$ & $\begin{array}{r}+62 \\
+72 \\
+133\end{array}$ & $\begin{array}{l}+38 \\
+36 \\
+37\end{array}$ \\
\hline 5 & $\begin{array}{r}8 \\
11 \\
19 \\
\end{array}$ & $\begin{array}{l}4 \\
2 \\
6 \\
\end{array}$ & $\begin{array}{l}22 \\
26 \\
48 \\
\end{array}$ & $\begin{array}{l}17 \\
21 \\
38 \\
\end{array}$ & $\begin{array}{l}29 \\
38 \\
67 \\
\end{array}$ & $\begin{array}{r}+55 \\
+73 \\
+128 \\
\end{array}$ & $\begin{array}{l}+34 \\
+36 \\
+35 \\
\end{array}$ \\
\hline 6 & $\begin{array}{r}8 \\
11 \\
19\end{array}$ & $\begin{array}{l}4 \\
2 \\
6\end{array}$ & $\begin{array}{l}22 \\
25 \\
47\end{array}$ & $\begin{array}{l}19 \\
21 \\
40\end{array}$ & $\begin{array}{l}30 \\
37 \\
67\end{array}$ & $\begin{array}{r}+59 \\
+71 \\
+130\end{array}$ & $\begin{array}{l}+36 \\
+35 \\
+36\end{array}$ \\
\hline 7 & $\begin{array}{r}8 \\
11 \\
19\end{array}$ & $\begin{array}{l}4 \\
3 \\
7\end{array}$ & $\begin{array}{l}21 \\
26 \\
47\end{array}$ & $\begin{array}{l}17 \\
22 \\
39\end{array}$ & $\begin{array}{l}31 \\
36 \\
67\end{array}$ & $\begin{array}{r}+59 \\
+69 \\
+128\end{array}$ & $\begin{array}{l}+36 \\
+34 \\
+35\end{array}$ \\
\hline 8 & $\begin{array}{r}7 \\
11 \\
18\end{array}$ & $\begin{array}{l}4 \\
1 \\
5\end{array}$ & $\begin{array}{l}22 \\
26 \\
48\end{array}$ & $\begin{array}{l}19 \\
21 \\
40\end{array}$ & $\begin{array}{l}30 \\
37 \\
67\end{array}$ & $\begin{array}{r}+61 \\
+72 \\
+133\end{array}$ & $\begin{array}{l}+37 \\
+36 \\
+37\end{array}$ \\
\hline 9 & $\begin{array}{r}9 \\
9 \\
18\end{array}$ & $\begin{array}{l}3 \\
4 \\
7\end{array}$ & $\begin{array}{l}20 \\
26 \\
46\end{array}$ & $\begin{array}{l}18 \\
21 \\
39\end{array}$ & $\begin{array}{l}31 \\
36 \\
67\end{array}$ & $\begin{array}{r}+58 \\
+71 \\
+129\end{array}$ & $\begin{array}{l}+36 \\
+35 \\
+36\end{array}$ \\
\hline 10 & $\begin{array}{r}8 \\
10 \\
18\end{array}$ & $\begin{array}{l}4 \\
3 \\
7\end{array}$ & $\begin{array}{l}22 \\
26 \\
48\end{array}$ & $\begin{array}{l}19 \\
21 \\
40\end{array}$ & $\begin{array}{l}30 \\
36 \\
66 \\
\end{array}$ & $\begin{array}{r}+59 \\
+70 \\
+129 \\
\end{array}$ & $\begin{array}{l}+36 \\
+35 \\
+36 \\
\end{array}$ \\
\hline 11 & $\begin{array}{r}9 \\
10 \\
19\end{array}$ & $\begin{array}{l}3 \\
2 \\
5\end{array}$ & $\begin{array}{l}21 \\
26 \\
47\end{array}$ & $\begin{array}{l}18 \\
21 \\
39\end{array}$ & $\begin{array}{l}29 \\
36 \\
67\end{array}$ & $\begin{array}{r}+55 \\
+70 \\
+126\end{array}$ & $\begin{array}{l}+34 \\
+35 \\
+35\end{array}$ \\
\hline 12 & $\begin{array}{r}9 \\
10 \\
19\end{array}$ & $\begin{array}{l}3 \\
2 \\
5\end{array}$ & $\begin{array}{l}21 \\
26 \\
47\end{array}$ & $\begin{array}{l}18 \\
21 \\
39\end{array}$ & $\begin{array}{l}29 \\
36 \\
67\end{array}$ & $\begin{array}{r}+54 \\
+71 \\
+125\end{array}$ & $\begin{array}{l}+33 \\
+35 \\
+35\end{array}$ \\
\hline 13 & $\begin{array}{r}8 \\
10 \\
18\end{array}$ & $\begin{array}{l}4 \\
3 \\
7\end{array}$ & $\begin{array}{l}22 \\
24 \\
46\end{array}$ & $\begin{array}{l}17 \\
21 \\
38\end{array}$ & $\begin{array}{l}29 \\
38 \\
67\end{array}$ & $\begin{array}{r}+55 \\
+74 \\
+129\end{array}$ & $\begin{array}{l}+34 \\
+37 \\
+36\end{array}$ \\
\hline 14 & $\begin{array}{r}9 \\
10 \\
19\end{array}$ & $\begin{array}{l}3 \\
2 \\
5\end{array}$ & $\begin{array}{l}22 \\
25 \\
47\end{array}$ & $\begin{array}{l}19 \\
21 \\
40\end{array}$ & $\begin{array}{l}30 \\
36 \\
66\end{array}$ & $\begin{array}{r}+57 \\
+71 \\
+128\end{array}$ & $\begin{array}{l}+35 \\
+35 \\
+35\end{array}$ \\
\hline 15 & $\begin{array}{r}8 \\
10 \\
18\end{array}$ & $\begin{array}{l}4 \\
3 \\
7\end{array}$ & $\begin{array}{l}22 \\
26 \\
48\end{array}$ & $\begin{array}{l}19 \\
21 \\
40\end{array}$ & $\begin{array}{l}30 \\
37 \\
67\end{array}$ & $\begin{array}{r}+59 \\
+72 \\
+131\end{array}$ & $\begin{array}{l}+36 \\
+36 \\
+36\end{array}$ \\
\hline TOTAL & $\begin{array}{l}120 \\
164 \\
284\end{array}$ & $\begin{array}{l}56 \\
38 \\
94\end{array}$ & $\begin{array}{l}324 \\
384 \\
708\end{array}$ & $\begin{array}{l}273 \\
316 \\
589\end{array}$ & $\begin{array}{l}450 \\
548 \\
998\end{array}$ & $\begin{array}{r}+877 \\
+1046 \\
+1923\end{array}$ & $\begin{array}{l}+36 \\
+35 \\
+36\end{array}$ \\
\hline
\end{tabular}


parables en nuestro sistema educativo, encontrando en todos los casos una clara actitud positiva independiente de la variable sexo.

Como hicimos en la Sección anterior, con el objetivo de realizar una comparación más directa entre los resultados de ambas técnicas de medida, usamos nuestra «escala de puntuaciones», adaptada para cada una de las técnicas. Los resultados se recogen en la tabla VI. Con ambas técnicas encontramos que alrededor del $56 \%$ (54\% usando la de DS y 59\% usando la de Likert) presenta una actitud positiva, y alrededor de un $12 \%$ ( $11 \%$ con DS y $14 \%$ con Likert), una actitud negativa. Estos resultados son semejantes a los obtenidos por alumnos de $2^{\circ}$ de BUP en 1991 (Espinosa y Román, 1991), pero ligeramente más positivos y, como era de esperar, son menos positivos que en estudiantes universitarios, aunque no tanto como cabía esperar por la variable optatividad que se supone en los universitarios.

\section{CONCLUSIONES}

En este trabajo hemos realizado una comparación entre dos de las más populares técnicas para evaluar las actitudes hacia las ciencias experimentales: el método de clases sumadas, generalmente conocido como escala de Likert; y el método de diferencial semántico. El estudio se llevó a cabo con estudiantes universitarios de $3^{\circ}$ de Químicas y estudiantes de enseñanza secundaria de $4^{\circ}$ de ESO, encontrando que ambas técnicas de evaluación de actitudes proporcionan resultados equiparables en nuestro sistema educativo. Este resultado es especialmente interesante por la simplicidad de la elaboración de los cuestionarios de diferencial semántico frente a los de Likert, y confirma en cierta medida los resultados obtenidos por nuestro grupo de investigación en trabajos previos usando la técnica de diferencial semántico, tanto en niveles universitarios como de enseñanza secundaria. Por último, hemos evaluado cualitativamente la influencia de la variable sexo, no encontrando diferencias significativas con ambas técnicas de medida.

Tabla VI

\begin{tabular}{|l|l|c|c|c|c|c|c|}
\cline { 3 - 9 } \multicolumn{2}{c|}{} & \multicolumn{2}{c|}{ VARONES } & \multicolumn{2}{c|}{ MUJERES } & \multicolumn{2}{c|}{ TOTALES } \\
\hline ESCALA & VALORACIÓN & DS & L & DS & L & DS & L \\
\hline $0-3$ o D+TD & Negativa & 11 & 12 & 10 & 14 & 11 & 14 \\
\hline $4-6$ o N & Indiferente & 32 & 27 & 37 & 26 & 35 & 27 \\
\hline $7-10$ o A+TA & Positiva & 55 & 60 & 52 & 58 & 54 & 59 \\
\hline
\end{tabular}

\section{REFERENCIAS BIBLIOGRÁFICAS}

ACEVEDO, J.A. (1993). IV Congreso sobre la Investigación en la Didáctica de las Ciencias y las Matemáticas. Barcelona, septiembre.

ARMAS, F., ESPINOSA, J. y ROMAN, T. (1996). Enseñanza de las Ciencias. Pendiente de publicación.

BRATT, M. (1984). Journal of Research in Science Teaching, 21, pp. 951-952.

EDWARDS, A.L. (1957). Techniques of attitude scale construction. Nueva York.

ESPINOSA, J. y ROMAN, T. (1991). Enseñanza de las Ciencias, 9, pp. 151-154.

ESPINOSA, J. y ROMAN, T. (1993). Enseñanza de las Ciencias, 11, pp. 297-300.

ESPINOSA, J. y ROMAN, T. (1995). Enseñanza de las Ciencias, 13, pp. 199-202.

GERMANN,P.J.(1988).Journal of Research in Science Teaching, 25, pp. 689-703.

HENERSON, E. (1985). Padres y maestros, 111, pp. 35-38.

LIKERT, R. (1934). Archives of Psychology, 140, pp. 1-55.
McCALLON, E.L. y BROWN, J.D. (1971). Journal of Experimental Education, 39, pp. 69-72.

MEJÍAS, L.A. (1988). Validation of a Spanish version of the Revised Science Attitude Scale for children with seventh grade students in Puerto Rico. Tesis doctoral no publicada. The Pennsylvania State University.

MISITI, F.L., Jr., SHRIGLEY, R.L. y HANSON, L. (1991). Science Education, 75, p. 525-540.

MUNBY, H. (1983). Journal of Research in Science Teaching, 20, pp. 141-162.

SCHIBECI, R.A. (1982). Science Education, 66, pp. 565-570.

SCHOFIELD, H.R. y START, K.B. (1978). Australian Journal of Education, 22, pp. 78-82.

SHRIGLEY, R.L. y KOBALLA T.R. Jr. (1984). Journal of Research in Science Teaching, 21, pp. 111-118.

SIMPSON, R.D. y OLIVER, J.S. (1985). Science Education, 69, pp. 511-526.

ZEIDLER, D.L.(1984). Journal of Research in Science Teaching, 21, pp. 341-342.

[Artículo recibido en abril de 1996 y aceptado en octubre de 1997.] 


\section{ANEXO I \\ CUESTIONARIO DE DIFERENCIAL SEMÁNTICO}

Has elegido una carrera en la que las asignaturas de ciencias son fundamentales.

A continuación se te expresan una serie de adjetivos o situaciones contrarias. Señala con una puntuación entre 0 y 10 cada una de ellas. Si crees que la cuestión se identifica con lo expuesto a la izquierda redondea el 10, si con la derecha el 0 . Si es una situación intermedia, según su proximidad a una u otra situación, acércate más o menos en la puntuación a uno de los extremos.

Sexo: $\quad$ Curso: $\quad$ Edad:
1. INTERESANTE
2. CLARO
3. FÁCIL
4. ME HA ACERCADO
A LA NATURALEZA
5. PROBARÍA OTRA VEZ
6. ASEQUIBLE
7. HE APRENDIDO
8. ME HA GUSTADO
9. ME HE DIVERTIDO
10. VALÍA LA PENA

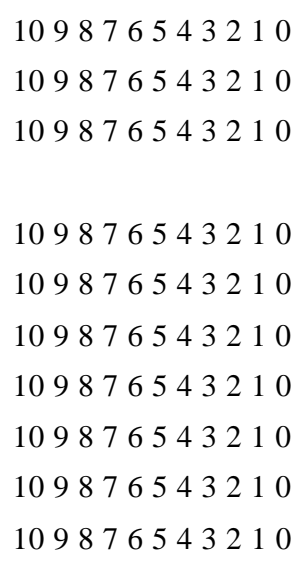

109876543210
109876543210

109876543210

109876543210

43210

109876543210

109876543210

109876543210
ABURRIDO
LIOSO
DIFÍCIL

¡NI EN BROMA!

JAMÁS

IMPOSIBLE

NADA

NADA

HE SUFRIDO

TIEMPO PERDIDO

\section{ANEXO II \\ CUESTIONARIO DE LIKERT}

Has elegido una carrera en la que las asignaturas de ciencias son fundamentales.

Queremos conocer tus actitudes y opiniones hacia la ciencia. Por favor, completa el siguiente cuestionario, donde TA es «totalmente de acuerdo»; $A$ es «de acuerdo»; $N$ es «ni fu ni fa»; $D$ es «en desacuerdo»; y $T D$ es «totalmente en desacuerdo».

$$
\text { Sexo: } \quad \text { Curso: } \quad \text { Edad: }
$$

1. Comprar libros de ciencias es pesadísimo

2. Odio pasar las notas de los experimentos al cuaderno

3. Las películas de ciencias me aburren mucho

4. Me gustaría que las clases de ciencias durasen todo el día

5. Odio las clases de ciencias

6. Me gustaría pertenecer a un club de ciencias que se reuniese después de las clases

7. Trabajar en el laboratorio no es mi idea de diversión

8. Me encuentro muy bien durante las clases de ciencias

9. Odio estudiar ciencias fuera de clase

10. No pienso hablar de ciencia con los amigos fuera de clase

11. Disfruto usando las matemáticas en los experimentos de ciencias

12. Hacer proyectos de ciencias en casa es estúpido

13. No me gustan los reportajes de ciencias en televisión

14. Aprender cosas de ciencias es pesadísimo

15. No tengo inconveniente en repetir un experimento varias veces para comprobar la respuesta

$$
\begin{array}{ccccc}
\text { TA } & \text { A } & \text { N } & \text { D } & \text { TD } \\
- & - & - & - & - \\
- & - & - & - & - \\
- & - & - & - & - \\
- & - & - & - & - \\
- & - & - & - & -
\end{array}
$$

